\title{
Capturing Greater Context for Question Generation
}

\author{
Luu Anh Tuan, ${ }^{*}$ Darsh J Shah, ${ }^{*}$ Regina Barzilay \\ Computer Science and Artificial Intelligence Lab, MIT \\ \{tuanluu,darsh,regina\}@csail.mit.edu
}

\begin{abstract}
Automatic question generation can benefit many applications ranging from dialogue systems to reading comprehension. While questions are often asked with respect to long documents, there are many challenges with modeling such long documents. Many existing techniques generate questions by effectively looking at one sentence at a time, leading to questions that are easy and not reflective of the human process of question generation. Our goal is to incorporate interactions across multiple sentences to generate realistic questions for long documents. In order to link a broad document context to the target answer, we represent the relevant context via a multi-stage attention mechanism, which forms the foundation of a sequence to sequence model. We outperform stateof-the-art methods on question generation on three questionanswering datasets - SQuAD, MS MARCO and NewsQA. ${ }^{1}$
\end{abstract}

\section{Introduction}

The tremendous popularity of reading comprehension through datasets like SQuAD (Rajpurkar et al. 2016), MS MARCO (Bajaj et al. 2016) and NewsQA (Trischler et al. 2016) has led to a surge in machine reading and reasoning techniques. These datasets are typically constructed using crowd sourcing which provides good questions but at a high cost of manual labor. There is an urgent need for automated methods to generate quality question-answer pairs from textual corpora.

Our goal is to generate a question for a target answer - a span of text in a provided document. To this end, we must be able to identify the relevant context for the question-answer from the document. Modeling long documents, however, is formidable, and our task involves understanding the relation between the answer and encompassing paragraphs, before asking the relevant question. Typically most existing methods have simplified the task by looking at just the answer containing sentence. However, this does not represent the human process of generating questions from a document.

\footnotetext{
* denotes equal contribution.

Copyright (c) 2020, Association for the Advancement of Artificial Intelligence (www.aaai.org). All rights reserved.

${ }^{1}$ Our code and data are available at https://github.com/vivisimo/ QuestionGeneration
}

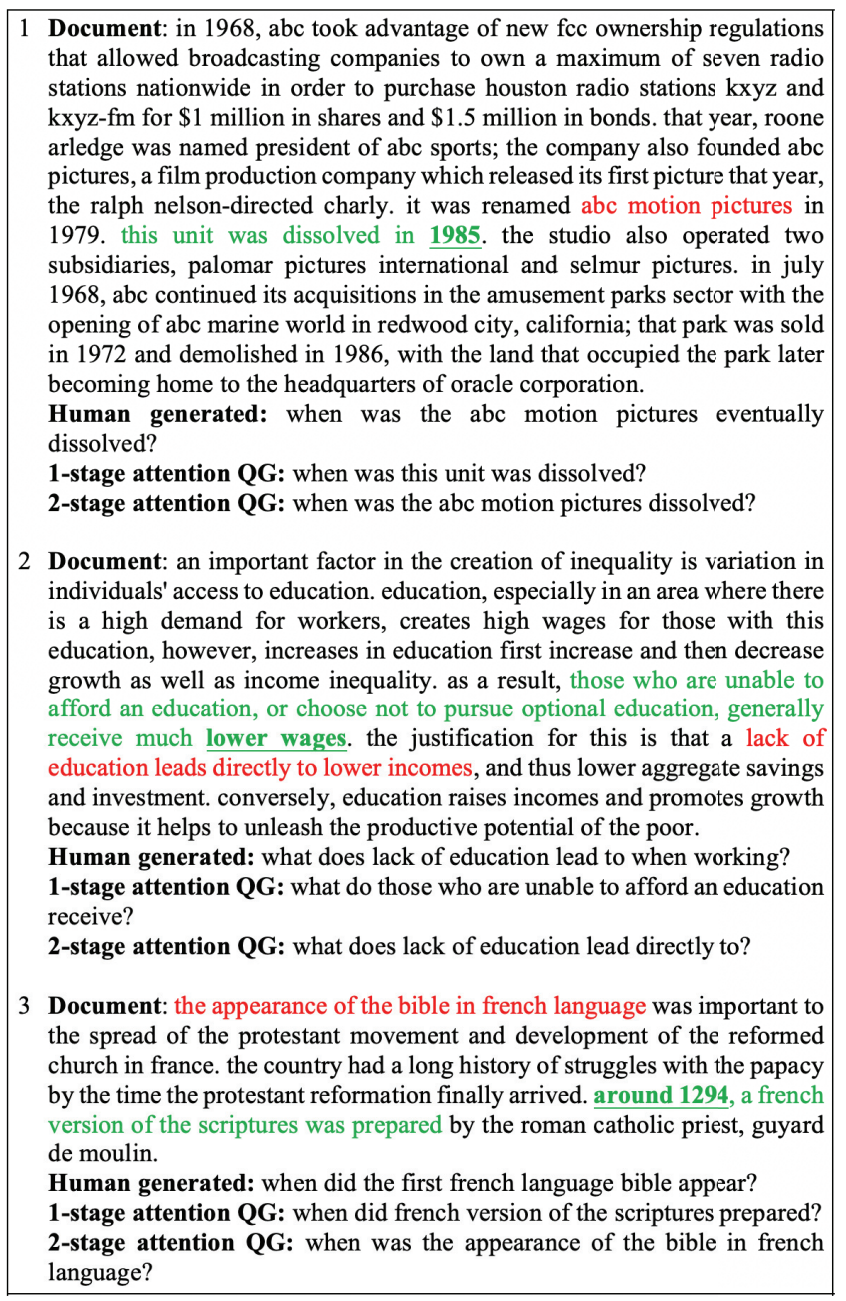

Figure 1: Examples (lower-cased) where multi-sentence context is required to ask the correct questions. Sentences containing answers are in green, while answers are underlined. The red phrases indicate additional background used by a human to generate the question. 1-stage and 2-stage attention QG are results generated by our model with different numbers of attention stages. 
For instance, crowd workers for the SQuAD dataset, as illustrated in Figure 1, used multiple sentences to ask a relevant question. In fact, as pointed out by (Du, Shao, and Cardie 2017), around $30 \%$ of the human-generated questions in SQuAD rely on information beyond a single sentence.

To accommodate such phenomenon, we propose a novel approach for document-level question generation by explicitly modeling the context based on a multi-stage attention mechanism. First, our method captures the immediate context, by attending the entire document with the answer to highlight phrases, e.g. "the unit was dissolved in" from example 1 in Figure 1, having direct relationship with the answer, i.e. " 1985 ". Next, by attending the original document representation with the attended document computed in the previous stage, we expand this context to include more phrases, e.g. "abc motion pictures", that have indirect relationship with the answer. We can repeat this process multiple times to increase the linkage-level of the answer-related background.

The final document representation, contains relevant answer context cues by means of attention weights. Through a copy-generate decoding mechanism, where at each step a word is either copied from the input or generated from the vocabulary, the attention weights guide the generation of the context words to produce high quality questions. The entire framework, from context collection to copy-generate style generation is trained end-to-end.

Our framework for document context representation, strengthened by more attention stages leads to a better question generation quality. Specifically, on SQuAD we get an absolute 5.79 jump in the Rouge points by using a second stage answer-attended representation of the document, compared to directly using the representation right after the first stage. We evaluate our hypothesis of using a controllable context to generate questions on three different QA datasets - SQuAD, MS MARCO, and NewsQA. Our method strongly outperforms existing state-of-the-art models by an average absolute increase of 1.56 Rouge, $0.97 \mathrm{Me}-$ teor and 0.81 Bleu scores over the previous best reported results on all three datasets.

\section{Related Work}

Question generation has been extensively studied in the past with broadly two main approaches, rule-based and learningbased.

Rule-based techniques These approaches usually rely on rules and templates of sentences' linguistic structures, and apply heuristics to generate questions (Chali and Hasan 2015; Heilman 2011; Lindberg et al. 2013; Labutov, Basu, and Vanderwende 2015). This requires human effort and expert knowledge, making scaling the approach very difficult. Neural methods tend to outperform and generalize better than these techniques.

Neural-based models Since (Serban et al. 2016; Du, Shao, and Cardie 2017), there have been many neural sequenceto-sequence models proposed for question generation tasks. These models are trained in an end-to-end manner and exploit the corpora of the question answering datasets to out- perform rule based methods in many benchmarks. However, in these initial approaches, there is no indication about parts of the document that the decoder should focus on in order to generate the question.

To generate a question for a given answer, (Subramanian et al. 2017; Kim et al. 2018; Zhou et al. 2017; Sun et al. 2018) applied various techniques to encode answer location information into an annotation vector corresponding to the word positions, thus allowing for better quality answerfocused questions. (Yuan et al. 2017) combined both supervised and reinforcement learning in the training to maximize rewards that measure question quality. (Liu et al. 2019) presented a syntactic features based method to represent words in the document in order to decide what words to focus on while generating the question.

The above studies, only consider sentence-level question generation, i.e. looking at one document sentence at a time. Recently, (Du and Cardie 2018) proposed a method that incorporated coreference knowledge into the neural networks to better encode this linguistically driven connection across entities for document-level question generation. Unfortunately, this work does not capture other relationships like semantic similarity. As in example 2 of Figure 1, two semantic-related phrases "lower wages" and "lower incomes" are needed to be linked together to generate the desired question. (Zhao et al. 2018) proposed another document-level question generation where they apply a gated self-attention mechanism to encode contextual information. However, their self-attention over the entire document is very noisy, redundant and contains many encoded dependencies that are irrelevant.

\section{Problem Definition}

In this section, we define the task of question generation. Given the document D and the answer A, we are interested in generating the question $\bar{Q}$ that satisfies:

$$
\bar{Q}=\underset{Q}{\operatorname{argmax}} \operatorname{Prob}(Q \mid D, A)
$$

where the document $D$ is a sequence of $l_{D}$ words: $D=$ $\left\{x_{i}\right\}_{i=1}^{l_{D}}$, the answer $A$ of length $l_{A}$ must be a sub-span of $D$ : $A=\left\{x_{j}\right\}_{j=m}^{n}$, where $1 \leq m<n \leq l_{D}$, and the question $\bar{Q}$ is a well-formed sequence of $l_{Q}$ words: $\bar{Q}=\left\{y_{k}\right\}_{k=1}^{l_{Q}}$ that can be answered from $D$ using $A$. The generated words $y_{k}$ can be derived from the document words $\left\{x_{i}\right\}_{i=1}^{l_{D}}$ or from a vocabulary $V$.

\section{Model Architecture}

In this section, we describe our proposed model for question generation. The key idea of our model is to use a multi-stage attention mechanism to attend to the important parts of the document that are related to the answer, and use them to generate the question. Figure 2 shows the high level architecture of the proposed model.

\subsection{Input and Context Encoding}

The input representation for the document and its interaction with the answer are described as follows. 


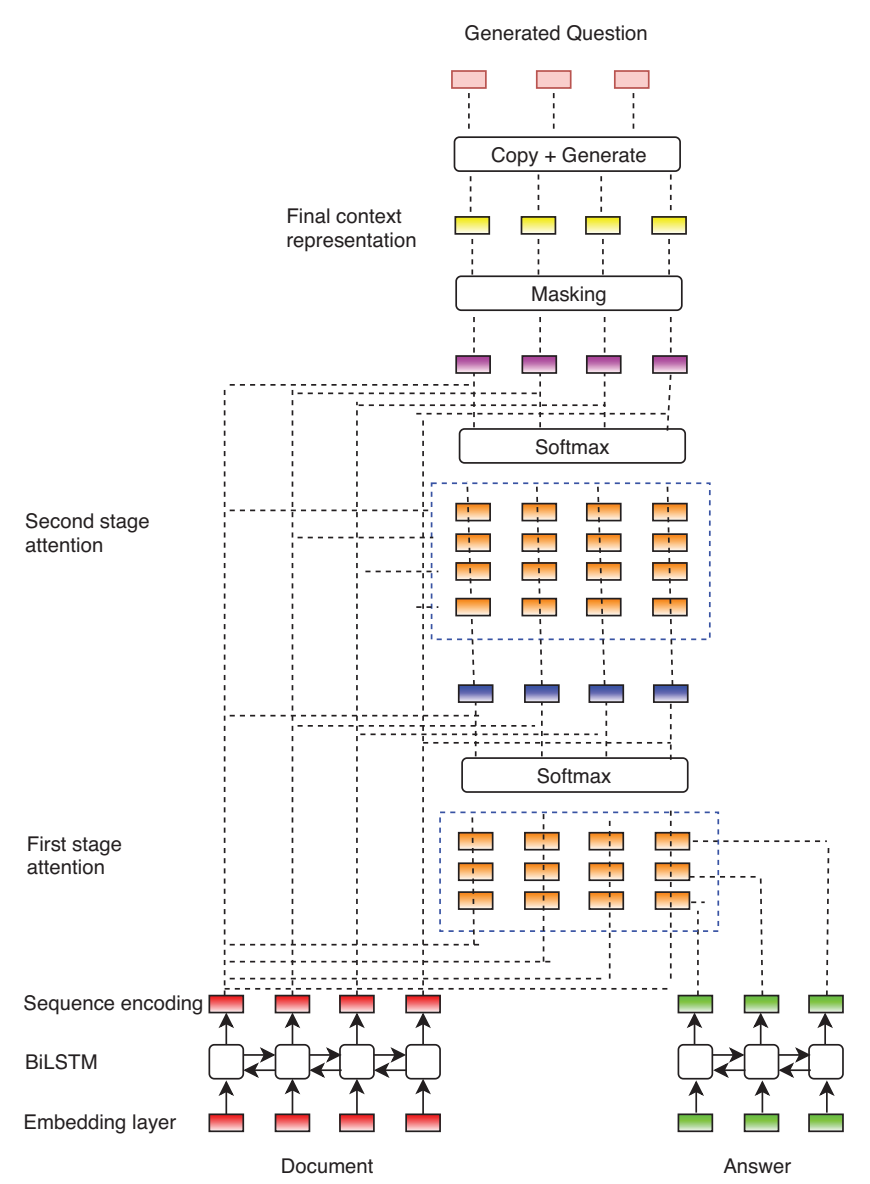

Figure 2: The architecture of our model (with two-stage attention). For simplicity we assume that the document has 4 words and the answer has 3 words.

Input Encoding Our model accepts two inputs, an answer $A$ and the document $D$ that the answer belongs to. Each of which is a sequence of words. The two sequences are indexed into a word embedding layer $W_{e m b}$ and then passed into a shared Bidirectional LSTM layer (Sak, Senior, and Beaufays 2014):

$$
\begin{aligned}
& H^{A}=\operatorname{BiLSTM}\left(\mathbf{W}_{\mathbf{e m b}}(A)\right) \\
& H^{D}=\operatorname{BiLSTM}\left(\mathbf{W}_{\mathbf{e m b}}(D)\right)
\end{aligned}
$$

where $H^{A} \in \mathbb{R}^{\ell_{A} \times d}$ and $H^{D} \in \mathbb{R}^{\ell_{D} \times d}$ are the hidden representations of $A$ and $D$ respectively, and $d$ is the hidden size of the Bidirectional LSTM.

Context Encoding The answer's context in the document is identified using our multi-stage attention mechanism, as described below.

Initial Stage (context with direct relation to answer): We pass $H^{D}, H^{A}$ into an alignment layer. Firstly, we compute a soft attention affinity matrix between $H^{D}$ and $H^{A}$ as follows:

$$
M_{i j}^{(1)}=\mathbf{F}\left(h_{i}^{D}\right) \mathbf{F}\left(h_{j}^{A}\right)^{\top}
$$

where $h_{i}^{D}$ is the $i^{t h}$ word in the document and $h_{j}^{A}$ is the $j^{t h}$ word in the answer. $\mathbf{F}(\cdot)$ is a standard nonlinear transformation function (i.e., $\mathbf{F}(x)=\sigma(\mathbf{W} x+\mathbf{b})$, where $\sigma$ indicates Sigmoid function), and is shared between the document and answer in this stage. $M^{(1)} \in \mathbb{R}^{\ell_{D} \times \ell_{A}}$ is the soft matching matrix. Next, we apply a column-wise max pooling of $M^{(1)}$. The key idea is to generate an attention vector:

$$
a^{(1)}=\operatorname{softmax}\left(\max _{\operatorname{col}}\left(M^{(1)}\right)\right)
$$

where $a^{(1)} \in \mathbb{R}^{l_{D}}$. Intuitively, each element $a_{i}^{(1)} \in a^{(1)}$ captures the degree of relatedness of the $i^{t h}$ word in document $D$ to answer $A$ based on its maximum relevance on each word of the answer. To learn the context sensitive weight importance of document, we then apply the attention vector on $H^{D}$ :

$$
C^{(1)}=H^{D} \odot a^{(1)}
$$

where $\odot$ denotes element-wise multiplication. $C^{(1)} \in R^{l_{D} \times d}$ can be considered as the first attended contextual representation of document where the words directly related to the answer are amplified with the high attention scores whilst the unrelated words are filtered out with low attention scores.

Iterative Stage (enhance the context with indirect relations): In this stage, we expand the context by collecting more words from the document that are related to directcontext computed in the first stage. We achieve this by attending the contextual attention representation of document obtained in stage 1 with original document representation as follows:

$$
\begin{aligned}
& M_{i j}^{(2)}=\mathbf{F}\left(h_{i}^{D}\right) \mathbf{F}\left(C_{j}\right)^{\top} \\
& a^{(2)}=\operatorname{softmax}\left(\max _{\operatorname{col}}\left(M^{(2)}\right)\right) \\
& C^{(2)}=H^{D} \odot a^{(2)}
\end{aligned}
$$

We can repeat the steps in this stage to enhance the context to the answer-related linkage level $k$. We denote the answerfocused context representation after $k$ stages as $C^{(k)}$. In our experiments, we train our models with a predefined value $k$, which is fine-tuned on the validation set.

Answer Masking Due to the enriched information in the context representation, it is essential for the model to know the position of the answer so that: (1) it can generate question that is coherent with the answer, and (2) does not include the exact answer in the question. We achieve this by masking the word representation at the position of the answer in the context representation $C^{(k)}$ with a special masking vector:

$$
C^{\mathrm{final}}=\operatorname{Mask}\left(C^{(k)}\right)
$$

$C^{\text {final }} \in R^{l_{D} \times d}$ can be considered as final contextual attention representation of document and will be used as the input to the decoder. 


\subsection{Decoding with Pointer Generator Network}

Using our context rich input representation $C^{\text {final }}$ computed previously, we move forward to the question generation. Our decoding framework is inspired by the pointer-generator network (See, Liu, and Manning 2017). The decoder is a BiLSTM, which at time-step $t$, takes as its input, the wordembedding of the previous time-step's output $W_{e}\left(y^{t-1}\right)$ and the latest decoder state attended input representation $r^{t}$ (described later in Equation (16)) to get the decoder state $h^{t}$ :

$$
h^{t}=\operatorname{BiLSTM}\left(\left[r^{t}, \mathbf{W}_{\mathbf{e}}\left(y^{t-1}\right)\right], h^{t-1}\right)
$$

Using the decoded state to generate the next word, where words can either be copied from the input; or generated by selecting from a fixed vocabulary:

$$
P_{\text {vocab }}=\operatorname{softmax}\left(\mathbf{V}^{\top}\left[h^{t}, r^{t}\right]\right)
$$

The generation probability $p_{\text {gen }} \in[0,1]$ at time-step $t$ depends on the context vector $r^{t}$, the decoder state $h^{t}$ and the decoder input $x^{t}=\left[r^{t}, \mathbf{W}_{\mathbf{e}}\left(y^{t-1}\right)\right]$ :

$$
p_{\text {gen }}=\sigma\left(\mathbf{w}_{\mathbf{r}} r^{t}+\mathbf{w}_{\mathbf{x}} x^{t}+\mathbf{w}_{\mathbf{h}} h^{t}\right)
$$

where $\sigma$ is the sigmoid function. This gating probability $p_{\text {gen }}$ is used to evaluate the probability of eliciting a word $w$ as follows:

$$
P(w)=p_{\text {gen }} P_{\text {vocab }}(w)+\left(1-p_{\text {gen }}\right) \sum_{i: w_{i}=w} a_{i}^{t}
$$

where $\sum_{i: w_{i}=w} a_{i}^{t}$ denotes the probability of word $w$ from the input being generated by the decoder:

$$
\begin{aligned}
e_{i}^{t} & =\mathbf{u}^{\top} \tanh \left(C_{i}^{\text {final }}+h^{t-1}\right) \\
a^{t} & =\operatorname{softmax}\left(e^{t}\right)
\end{aligned}
$$

Unlike traditional sequence to sequence models, our input $C^{\text {final }}$ is already weighted via the answer level self-attention. This weighting is reflected directly in the final generation via the copy mechanism through $a^{t}$, and is also used to evaluate the input context representation $r^{t}$ :

$$
r^{t}=\sum_{i} a_{i}^{t} C_{i}^{\mathrm{final}}
$$

Finally, the word output at time step $t, y^{t}$ is identified as:

$$
y^{t}=\underset{w}{\operatorname{argmax}} P(w)
$$

The model is trained in an end to end framework, to maximize the probability of generating the target sequence $y^{1}, \ldots, y^{l_{Q}}$. At each time step $t$, the probability of predicting $y^{t}$ is optimized using cross-entropy from the probability of words over the entire vocabulary (fixed and document words). Once the model is trained, we use beam search for inference during decoding. The beam search is parameterised by the possible number of paths $k$.

\section{Experimental Setup}

In this section we describe the experimental setting to study the proficiency of our proposed model.

\subsection{Datasets}

We evaluate our model on 3 question answering datasets: SQuAD (Rajpurkar et al. 2016), MS Marco (Bajaj et al. 2016) and NewsQA (Trischler et al. 2016). These form a comprehensive set of datasets to evaluate question generation.

SQuAD. SQuAD is a large scale reading comprehension dataset containing close to $100 \mathrm{k}$ questions posed by crowdworkers on a set of Wikipedia articles, where the answer is a span in the article. The dataset for our question generation task is constructed from the training and development set of the accessible parts of SQuAD. To be able to directly compare with other reported results, we consider the two following splits:

- Split1: similar to (Zhao et al. 2018), we keep the SQuAD train set and randomly split the SQuAD dev set into our dev and test set with the ratio 1:1. The split is done at sentence level.

- Split2: similar to (Du, Shao, and Cardie 2017), we randomly split the original SQuAD train set randomly into train and dev set with the ratio 9:1, and keep the SQuAD dev set as our test set. The split is done at article level.

MS MARCO. MS MARCO is the human developed question answering dataset derived from a million Bing search queries. Each query is associated with paragraphs from multiple documents resulting from Bing, and the dataset mentions the list of ground truth answers from these paragraphs. Similar to (Zhao et al. 2018), we extract a subset of MS Marco where the answers are sub-spans within the paragraphs, and then randomly split the original train set into train $(51 \mathrm{k})$ and $\operatorname{dev}(6 \mathrm{k})$ sets. We use the $7 \mathrm{k}$ questions from the original dev set as our test set.

NewsQA. NewsQA is the human generated dataset based on CNN news articles. Human crowd-workers are motivated to ask questions from headlines of the articles and the answers are found by other workers from the articles contents. In our experiment, we select the questions in NewsQA where answers are sub-spans within the articles. As a result, we obtain a dataset with $76 \mathrm{k}$ questions for train set, and $4 \mathrm{k}$ questions for each dev and test set.

Table 1 gives the details of the three datasets used in our experiments.

\begin{tabular}{|c|c|c|c|c|c|c|}
\hline Dataset & Train & Dev & Test & $l_{D}$ & $l_{Q}$ & $l_{A}$ \\
\hline SQuAD-1 & 87,488 & 5,267 & 5,272 & 126 & 11 & 3 \\
SQuAD-2 & 77,739 & 9,749 & 10,540 & 127 & 11 & 3 \\
MS Marco & 51,000 & 6,000 & 7,000 & 60 & 6 & 15 \\
NewsQA & 76,560 & 4,341 & 4,292 & 583 & 8 & 5 \\
\hline
\end{tabular}

Table 1: Description of the evaluation datasets. $l_{D}, l_{Q}$ and $l_{A}$ stand for average length of document, question and answer respectively.

\subsection{Implementation Details}

We use a one-layer Bidirectional LSTM with hidden dimension size of 512 for the encoder and decoder. Our en- 


\begin{tabular}{|l||c|c|c|c||c|c|}
\hline Model & Bleu-1 & Bleu-2 & Bleu-3 & Bleu-4 & Meteor & Rouge-L \\
\hline PCFG-Trans & 28.77 & 17.81 & 12.64 & 9.47 & 18.97 & 31.68 \\
SeqCopyNet & - & - & - & 13.02 & - & 44.00 \\
seq2seq+Z+c+GAN & 44.42 & 26.03 & 17.60 & 13.36 & 17.70 & 40.42 \\
NQG++ & 42.36 & 26.33 & 18.46 & 13.51 & 18.18 & 41.60 \\
MPQG & - & - & - & 13.91 & - & - \\
APM & 43.02 & 28.14 & 20.51 & 15.64 & - & - \\
ASs2s & - & - & - & 16.17 & - & - \\
S2sa-at-mp-gsa & 45.69 & 30.25 & 22.16 & 16.85 & 20.62 & 44.99 \\
CGC-QG & 46.58 & 30.90 & 22.82 & 17.55 & 21.24 & 44.53 \\
\hline Our model & $\mathbf{4 6 . 6 0}$ & $\mathbf{3 1 . 9 4}$ & $\mathbf{2 3 . 4 4}$ & $\mathbf{1 7 . 7 6}$ & $\mathbf{2 1 . 5 6}$ & $\mathbf{4 5 . 8 9}$ \\
\hline
\end{tabular}

Table 2: Results in question generation on SQuAD split1

\begin{tabular}{|l||c|c|c|c||c|c|}
\hline Model & Bleu-1 & Bleu-2 & Bleu-3 & Bleu-4 & Meteor & Rouge-L \\
\hline LTA & 43.09 & 25.96 & 17.50 & 12.28 & 16.62 & 39.75 \\
MPQG & - & - & - & 13.98 & 18.77 & 42.72 \\
CorefNQG & - & - & 20.90 & 15.16 & 19.12 & - \\
ASs2s & - & - & - & 16.20 & 19.92 & 43.96 \\
S2sa-at-mp-gsa & 45.07 & 29.58 & 21.60 & 16.38 & 20.25 & 44.48 \\
\hline Our model & $\mathbf{4 5 . 1 3}$ & $\mathbf{3 0 . 4 4}$ & $\mathbf{2 3 . 4 0}$ & $\mathbf{1 7 . 0 9}$ & $\mathbf{2 1 . 2 5}$ & $\mathbf{4 5 . 8 1}$ \\
\hline
\end{tabular}

Table 3: Results in question generation on SQuAD split2

tire model is trained end-to-end, with batch size 64, maximum of 200k steps, and Adam optimizer with a learning rate of 0.001 and $\mathrm{L} 2$ regularization set to $10^{-6}$. We initialize our word embeddings with frozen pre-trained GloVe vectors (Pennington, Socher, and Manning 2014). Text is lowercased and tokenized with NLTK. We tune the step of biattention used in encoder from $\{1,2,3\}$ on the development set. During decoding, we used beam search with the beam size of 10 , and stopped decoding when every beam in the stack generates the $<E O S>$ token.

\subsection{Evaluation}

Most of the prior studies evaluate the model performances against target questions using automatic metrics. In order to have an empirical comparison, we too use Bleu-1, Bleu-2, Bleu-3, Bleu-4 (Papineni et al. 2002), METEOR (Denkowski and Lavie 2014) and ROUGE-L (Lin 2004) to evaluate the question generation methods. Bleu measures the average n-gram precision on a set of reference sentences. METEOR is a recall-oriented metric used to calculate the similarity between generations and references. ROUGE-L is used to evaluate longest common sub-sequence recall of the generated sentences compared to references. A question structurally and syntactically similar to the human question would score high on these metrics, indicating relevance to the document and answer.

In order to have a more complete evaluation, we also report human evaluation results, where annotators evaluate the quality of questions generated on two important parameters: naturalness (grammar) and difficulty (in answering the question) (Section 6.2).

\subsection{Baselines}

As baselines, we compare our proposed model against several prior work on question generation. These include:

- PCFG-Trans (Heilman 2011): a rule-based system that generates a question based on a given answer word span.

- LTA (Du, Shao, and Cardie 2017): the seminal Seq2seq model for question generation.

- ASs2s (Kim et al. 2018): a Seq2Seq model learns to identify which interrogative word should be used by replacing the answer in the original passage with a special token.

- MPQG (Song et al. 2018): a Seq2Seq model that matches the answer with the passage before generating question

- QG+QA (Duan et al. 2017): a model that combines supervised and reinforcement learning for question generation

- NQG++ (Zhou et al. 2017): a Seq2Seq model with a feature-rich encoder to encode answer position, POS and NER tag information.

- APM (Sun et al. 2018): a model that incorporates the relative distance between the context words and answer when generating the question.

- S2sa-at-mp-gsa (Zhao et al. 2018) : a Seq2Seq model that uses gate self-attention and maxout-pointer mechanism to encode the context of question.

- SeqCopyNet (Zhou et al. 2018): a Seq2Seq model that use the copying mechanism to copy not only a single word but a sequence from the input sentence.

- Seq2seq+z+c+GAN (Yao et al. 2018): a GAN-based model captures the diversity and learning representation using the observed variables. 


\begin{tabular}{|l||c|c|c|c||c|c|}
\hline Model & Bleu-1 & Bleu-2 & Bleu-3 & Bleu-4 & Meteor & Rouge-L \\
\hline LTA & - & - & - & 10.46 & - & - \\
QG+QA & - & - & - & 11.46 & - & - \\
S2sa-at-mp-gsa & - & - & - & 17.24 & - & - \\
\hline Our model & $\mathbf{4 1 . 4 3}$ & $\mathbf{2 9 . 9 7}$ & $\mathbf{2 3 . 0 1}$ & $\mathbf{1 8 . 2 5}$ & $\mathbf{4 2 . 7 7}$ & $\mathbf{1 9 . 4 3}$ \\
\hline
\end{tabular}

Table 4: Results in question generation on MS MARCO

\begin{tabular}{|l||c|c|c|c||c|c|}
\hline Model & Bleu-1 & Bleu-2 & Bleu-3 & Bleu-4 & Meteor & Rouge-L \\
\hline PCFG-Trans & 16.90 & 7.94 & 4.72 & 3.08 & 13.74 & 23.78 \\
MPQG & 35.70 & 17.16 & 9.64 & 5.65 & 14.13 & 39.85 \\
NQG++ & 40.33 & 22.47 & 14.83 & 9.94 & 16.72 & 42.25 \\
CGC-QG & 40.45 & 23.52 & 15.68 & 11.06 & 17.43 & 43.16 \\
\hline Our model & $\mathbf{4 2 . 5 4}$ & $\mathbf{2 6 . 1 4}$ & $\mathbf{1 7 . 3 0}$ & $\mathbf{1 2 . 3 6}$ & $\mathbf{1 9 . 0 4}$ & $\mathbf{4 4 . 0 5}$ \\
\hline
\end{tabular}

Table 5: Results in question generation on NewsQA

- CorefNQG (Du and Cardie 2018): a Seq2Seq model that utilizes the coreference information to link the contexts.

- CGC-QG (Liu et al. 2019): a Seq2Seq model that learns to make decisions on which words to generate and to copy using rich syntactic features.

\section{Results and Analysis}

In this section, we discuss the experimental results and some ablation studies of our proposed model.

\subsection{Comparison with Baseline Models}

We present the question generation performance of baseline models and our model on the three QA datasets in Tables $2,3,4$ and $5^{2}$. We find that our model consistently outperforms all other baselines and sets a new state-of-the-art on all datasets and across different splits.

For SQuAD split-1, we achieve an average absolute improvement of 0.2 in Bleu-4, 0.3 in Meteor and 1.3 points in Rouge-L score compared to the best previous reported result. ${ }^{3}$ For SQuAD split-2, we achieve even higher average absolute improvement of $0.7,1.0$ and 1.4 points of Bleu4, Meteor and Rouge-L scores respectively, compared to S2sa-at-mp-gsa - the best previous model on the dataset and also a document-level question generation model. Showing that our model can identify better answer-related context for question generation compared to other document-level methods. On the MS MARCO dataset, where the ground truth questions are more natural, we achieve an absolute improvement of 1.0 in Bleu-4 score compared to the best previous reported result.

On the NewsQA dataset, which is the harder dataset as the length of input documents are very long, our overall perfor-

\footnotetext{
${ }^{2}$ For most baselines, we don't have access to their implementations. Hence, we present results for only datasets that they report on in their papers.

${ }^{3}$ We take 5 random splits and report the average across the splits. The lowest performance of the 5 runs also exceeds the stateof-the-art in this setting. Previous methods take an equal random split of the development set into dev/test sets. This can lead to inconsistencies in comparisons.
}

mance is still promising. Our model outperforms the CGCQG model by an average absolute score 1.3 of Bleu-4, 1.6 of Meteor, and 0.9 of Rouge-L, again demonstrating that exploiting the broader context can help the question generation system better match humans at the task.

\subsection{Human Evaluation}

To measure the quality of questions generated by our system, we conduct a human evaluation. Most of the previous work, except the LTA system (Du, Shao, and Cardie 2017), do not conduct any human evaluation, and for most of the competing methods, we do not have the code to reproduce the outputs. Hence, we conduct human evaluation using the exact same settings and metrics in (Du, Shao, and Cardie 2017) for a fair comparison. Specifically, we consider two criterion in human evaluation: (1) Naturalness, which indicates the grammaticality and fluency; and (2) Difficulty, which measures the syntactic divergence and the reasoning needed to answer the question. We randomly sample 100 sentence-question pairs from our SQuAD experimental outputs. We then ask four professional English speakers to rate the pairs in terms of the above criterion on a $1-5$ scale ( 5 for the best). The experimental result is given in Table 6 .

\begin{tabular}{|l|l|l|}
\hline & Naturalness & Difficulty \\
\hline LTA & 3.36 & 3.03 \\
Our model & 3.68 & $\mathbf{3 . 2 7}$ \\
\hline $\begin{array}{l}\text { Human generated ques- } \\
\text { tions }\end{array}$ & $\mathbf{4 . 0 6}$ & 2.65 \\
\hline
\end{tabular}

Table 6: Human evaluation results for question generation. Naturalness and difficulty are rated on a $1-5$ scale ( 5 for the best).

The inter-rater agreement of Krippendorff's Alpha between human evaluations is 0.21 . The results imply that our model can generate questions of better quality than the LTA system. Our system tends to generate difficult questions owing to the fact that it gathers context from the whole document rather than from just one or two sentences. 


\subsection{Ablation Study}

In this section, we study the impact of (1) The proposed attention mechanism in the encoder; (2) The number of attention stages used in that mechanism; and (3) The masking technique used for the encoder.

\begin{tabular}{|l||c|c|c|}
\hline Model & Bleu-4 & Meteor & Rouge-L \\
\hline Original (2-stage attention) & $\mathbf{1 7 . 7 6}$ & $\mathbf{2 1 . 5 6}$ & $\mathbf{4 5 . 8 9}$ \\
- without attention & 3.06 & 10.83 & 28.75 \\
- without masking & 5.19 & 13.08 & 31.14 \\
- with 1-stage attention & 14.52 & 18.28 & 40.10 \\
- with 3-stage attention & 12.87 & 16.05 & 38.33 \\
\hline
\end{tabular}

Table 7: Ablation study on SQuAD split 1.

Impact of using encoder attention In this ablation, we remove the attention mechanism in the encoder and just pass the vanilla document representation to the decoder. As shown in Table 7, without using attention mechanism, the performance drops significantly (more than 14 Bleu points). We hypothesize that without attention, the model lacks the capability to identify the import parts of document and hence generates questions unrelated to the target answer.

Impact of number of attention stages As shown in Table 7 , with an increase in the number of attention stages from 1 to 2 , the performance of model improves significantly, with an increment of more than 3 Bleu- 4 points.

To have a deeper understanding about the impact of the number of attention stages, we calculate for the words in the document that occurred in the ground truth question, their total attention score at the end of input attention layer as in Figure 3. For 1-stage and 2-stage attention, the total attention score of the question words to be copied from the document are 0.43 and 0.52 respectively, demonstrating that in SQuAD dataset, the 2-stage attention covers more of the question words in a focused manner. An example for this effect can be seen in Figure 4. The extra stage clearly helps in gathering more relevant context to generate a question closer to the ground-truth.

However, on further increasing the number of attention stages to 3 , we observe that the quality of generated questions deteriorates. This can be attributed to the fact that for most of the questions in $\mathrm{SQuAD}$, such 3-stage attention leads to a very cloudy context, where several words get covered, but with a diluted attention. 3-stage attention's coverage in Figure 3 shows this clearly, where its coverage in ground-truth questions is lower than even the 1-stage attention, justifying its poor question generation quality.

Impact of masking While attending to the answer's context and the related sentences is crucial, we find that it is imperative to mask out the answer before getting the input representation. It is demonstrated from the experimental results in Table 7 where the Bleu-4 score is increased more than 12 points when applying this masking.

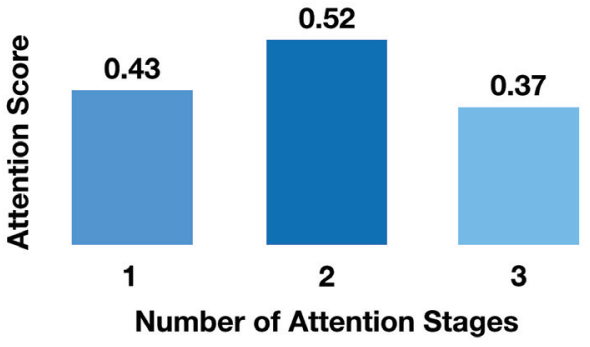

Figure 3: Average total attention score of words in the document that occurred in the ground truth question when using different attention stages (SQuAD split 1).

1-stage attention: the appearance of the bible in french language was important to the spread of the protestant movement and development of the reformed church in france. the country had a long history of struggles with the papacy by the time the protestant reformation finally arrived. around $\underline{1294}$, a french version of the scriptures was prepared by the roman catholic priest, guyard de moulin.

2-stage attention: the appearance of the bible in french language was important to the spread of the protestant movement and development of the reformed church in france. the country had a long history of struggles with the papacy by the time the protestant reformation finally arrived. around 1294, a french version of the scriptures was prepared by the roman catholic priest, guyard de moulin.

Human generated: when did the first french language bible appear? 1-stage attention QG: when did french version of the scriptures prepared? 2-stage attention QG: when was the appearance of the bible in french language?

Figure 4: Qualitative analysis of attention vector. The intensity of the color (red) denotes the strength of the attention weights.

\subsection{Case Study}

Figure 1 shows some examples that the document-level information obtained from our proposed multi-stage attention mechanism is needed to generate the correct questions.

In example 1, the two-stage attention model is able to identify the phrases "this unit" referring to "abc motion pictures", which is out of the sentence containing the answer.

In example 2, two semantic-related phrases "lower incomes" and "lower wages" in two different sentences are successfully linked by our two-stage attention model to generate the correct question.

In example 3, the two-stage attention model is able to link two different sentences containing the same word ("french") and semantic-related words ("bible" and "scriptures"), forming relevant context for generating the expected question.

\section{Conclusion}

In this paper, we proposed a novel document-level approach for question generation by using multi-step recursive attention mechanism on the document and answer representation to extend the relevant context. We demonstrate that taking additional attention steps helps learn a more relevant con- 
text, leading to a better quality of generated questions. We evaluate our method on three QA datasets - SQuAD, MS MARCO and NewsQA, and set the new state-of-the-art results in question generation for all of them.

\section{Acknowledgments}

We thank the MIT NLP group for their helpful discussion and comments. This work is supported by DSO grant DSOCL18002.

\section{References}

Bajaj, P.; Campos, D.; Craswell, N.; Deng, L.; Gao, J.; Liu, X.; Majumder, R.; McNamara, A.; Mitra, B.; Nguyen, T.; et al. 2016. Ms marco: A human generated machine reading comprehension dataset. arXiv preprint arXiv:1611.09268.

Chali, Y., and Hasan, S. A. 2015. Towards topic-to-question generation. Computational Linguistics 41(1):1-20.

Denkowski, M., and Lavie, A. 2014. Meteor universal: Language specific translation evaluation for any target language. In Proceedings of the ninth workshop on statistical machine translation, 376380.

Du, X., and Cardie, C. 2018. Harvesting paragraph-level questionanswer pairs from wikipedia. In Proceedings of the 56th Annual Meeting of the Association for Computational Linguistics (Volume 1: Long Papers), 1907-1917.

Du, X.; Shao, J.; and Cardie, C. 2017. Learning to ask: Neural question generation for reading comprehension. In Proceedings of the 55th Annual Meeting of the Association for Computational Linguistics (Volume 1: Long Papers), 1342-1352.

Duan, N.; Tang, D.; Chen, P.; and Zhou, M. 2017. Question generation for question answering. In Proceedings of the 2017 Conference on Empirical Methods in Natural Language Processing, 866-874.

Heilman, M. 2011. Automatic factual question generation from text. In Language Technologies Institute, School of Computer Science, Carnegie Mellon University.

Kim, Y.; Lee, H.; Shin, J.; and Jung, K. 2018. Improving neural question generation using answer separation. In Thirty-Third AAAI Conference on Artificial Intelligence.

Labutov, I.; Basu, S.; and Vanderwende, L. 2015. Deep questions without deep understanding. In Proceedings of the 53rd Annual Meeting of the Association for Computational Linguistics and the 7th International Joint Conference on Natural Language Processing, 889-898.

Lin, C.-Y. 2004. Rouge: A package for automatic evaluation of summaries. Text Summarization Branches Out.

Lindberg, D.; Popowich, F.; Nesbit, J.; and Winne, P. 2013. Generating natural language questions to support learning on-line. In Proceedings of the 14th European Workshop on Natural Language Generation, 105-114.

Liu, B.; Zhao, M.; Niu, D.; Lai, K.; He, Y.; Wei, H.; and Xu, Y. 2019. Learning to generate questions by learningwhat not to generate. In The World Wide Web Conference, 1106-1118. ACM.

Papineni, K.; Roukos, S.; Ward, T.; and Zhu, W.-J. 2002. Bleu: a method for automatic evaluation of machine translation. In Proceedings of the 40th annual meeting on association for computational linguistics, 311-318. Association for Computational Linguistics.

Pennington, J.; Socher, R.; and Manning, C. 2014. Glove: Global vectors for word representation. In Proceedings of the 2014 conference on empirical methods in natural language processing (EMNLP), 1532-1543.

Rajpurkar, P.; Zhang, J.; Lopyrev, K.; and Liang, P. 2016. Squad: $100,000+$ questions for machine comprehension of text. In Proceedings of the 2016 Conference on Empirical Methods in Natural Language Processing, 2383-2392.

Sak, H.; Senior, A.; and Beaufays, F. 2014. Long short-term memory recurrent neural network architectures for large scale acoustic modeling. In Fifteenth annual conference of the international speech communication association.

See, A.; Liu, P. J.; and Manning, C. D. 2017. Get to the point: Summarization with pointer-generator networks. In Proceedings of the 55th Annual Meeting of the Association for Computational Linguistics (Volume 1: Long Papers), 1073-1083. Vancouver, Canada: Association for Computational Linguistics.

Serban, I. V.; García-Durán, A.; Gulcehre, C.; Ahn, S.; Chandar, S.; Courville, A.; and Bengio, Y. 2016. Generating factoid questionswith recurrent neural networks: The $30 \mathrm{~m}$ factoid question-answer corpus. In 54th Annual Meeting of the Association for Computational Linguistics, ACL 2016, 588-598.

Song, L.; Wang, Z.; Hamza, W.; Zhang, Y.; and Gildea, D. 2018. Leveraging context information for natural question generation. In Proceedings of the 2018 Conference of the North American Chapter of the Association for Computational Linguistics: Human Language Technologies, 569-574.

Subramanian, S.; Wang, T.; Yuan, X.; Zhang, S.; Bengio, Y.; and Trischler, A. 2017. Neural models for key phrase detection and question generation. arXiv preprint arXiv:1706.04560.

Sun, X.; Liu, J.; Lyu, Y.; He, W.; Ma, Y.; and Wang, S. 2018. Answer-focused and position-aware neural question generation. In Proceedings of the 2018 Conference on Empirical Methods in Natural Language Processing, 3930-3939.

Trischler, A.; Wang, T.; Yuan, X.; Harris, J.; Sordoni, A.; Bachman, P.; and Suleman, K. 2016. Newsqa: A machine comprehension dataset. arXiv preprint arXiv:1611.09830.

Yao, K.; Zhang, L.; Luo, T.; Tao, L.; and Wu, Y. 2018. Teaching machines to ask questions. In Proceedings of the 27th International Joint Conference on Artificial Intelligence, 4546-4552. AAAI Press.

Yuan, X.; Wang, T.; Gulcehre, C.; Sordoni, A.; Bachman, P.; Zhang, S.; Subramanian, S.; and Trischler, A. 2017. Machine comprehension by text-to-text neural question generation. In Proceedings of the 2nd Workshop on Representation Learning for NLP, $15-25$.

Zhao, Y.; Ni, X.; Ding, Y.; and Ke, Q. 2018. Paragraph-level neural question generation with maxout pointer and gated self-attention networks. In Proceedings of the 2018 Conference on Empirical Methods in Natural Language Processing, 3901-3910.

Zhou, Q.; Yang, N.; Wei, F.; Tan, C.; Bao, H.; and Zhou, M. 2017. Neural question generation from text: A preliminary study. In $\mathrm{Na}$ tional CCF Conference on Natural Language Processing and Chinese Computing, 662-671. Springer.

Zhou, Q.; Yang, N.; Wei, F.; and Zhou, M. 2018. Sequential copying networks. In Thirty-Second AAAI Conference on Artificial Intelligence. 